\title{
Comparison of Plain Balloon and Cutting Balloon Angioplasty for the Treatment of Restenosis With Drug-Eluting Stents vs Bare Metal Stents
}

\author{
Seung-Jung Park, MD; Kyung-Hee Kim, MD; Il-Young Oh, MD; Dong-Ho Shin, MD; \\ Kyung-Il Park, MD; Myung-Ki Seo, MD; Jin-Wook Chung, MD; Kyung Woo Park, MD; \\ Hae-Young Lee, MD; Hyun-Jae Kang, MD; Bon-Kwon Koo, MD; \\ Tae-Jin Youn, MD; Hyo-Soo Kim, MD
}

\begin{abstract}
Background: The efficacy of cutting or plain balloon angioplasty (CBA or PBA) has not been analyzed for the treatment of drug-eluting stent (DES) restenosis vs bare metal stent (BMS) restenosis.

Methods and Results: The 252 in-stent restenosis (ISR) lesions in 224 consecutive patients treated by CBA $(n=167)$ or PBA $(n=85)$ between July 2002 and December 2007 were analyzed. At 6-month angiographic and 12-month clinical follow-up, CBA and PBA showed similar efficacies: repeat-ISR ( $37.0 \%$ vs $37.8 \%, P=0.90)$ ), late loss $(0.62 \pm 0.60$ vs $0.61 \pm 0.47 \mathrm{~mm}, P=0.92)$, and target lesion revascularization ( $18.3 \%$ vs $22.4 \%, P=0.50)$. This comparable efficacy was maintained for treatment in the DES-ISR and BMS-ISR subgroups. However, target lesion-related myocardial infarction $(n=9)$ occurred more frequently in the CBA than in the PBA arm $(6.2 \%$ vs $0 \%$, $P=0.03)$, most of which developed early after ISR treatment ( $n=7 ; 54 \pm 26$ days). Independent predictors of repeatISR were diffuse ISR and smaller pretreatment minimal lumen diameter, both of which might imply heavier plaque burden in the ISR group.
\end{abstract}

Conclusions: Plain or cutting balloon angioplasty for ISR seems to be comparable, as the angiographic or clinical endpoints were not affected by initial stent type but by parameters related to the plaque burden of the ISR lesion. However, CBA might be associated with higher risk of myocardial infarction than PBA, suggesting more attention to dual-antiplatelet therapy after its use for ISR. (Circ $J$ 2010; 74: 1837-1845)

Key Words: Bare metal stent; Cutting balloon angioplasty; Drug-eluting stent; In-stent restenosis; Plain balloon angioplasty

$\mathbf{T}$ he risk of restenosis has significantly decreased with the advent of drug-eluting stents (DES). ${ }^{1,2}$ However, restenosis rates have been as high as $20-30 \%$ in complex lesions despite the use of DES..$^{3-5}$ In addition, substantial percentages of patients are still treated with bare metal stents (BMS), which are associated with a higher incidence of restenosis than DES.

\section{Editorial $\mathrm{p} 1796$}

Optimal management of in-stent restenosis (ISR), however, has not been definitively established. ${ }^{6-8}$ Additional DES implantation for ISR poses vexing safety issues, such as stent thrombosis (STH), ${ }^{9-11}$ and brachytherapy or atherectomy is technically very cumbersome and not feasible in all institu- tions. ${ }^{12,13}$ On the other hand, balloon angioplasty, in terms of convenience, cost-effectiveness, and safety issue could still be an attractive approach for the treatment of ISR.

For the treatment of patients with de novo coronary lesions or BMS-ISR, the superiority of cutting balloon angioplasty (CBA) over plain balloon angioplasty (PBA) has been suggested by some initial reports. ${ }^{14,15}$ But these early results have not been reproduced in larger randomized trials. ${ }^{16,17}$ For the problem of DES-ISR, there is little data comparing the efficacy of CBA and PBA. Therefore, the purpose of this study was firstly to compare the efficacy of CBA and PBA in the treatment of DES-ISR vs BMS-ISR and secondly to determine the independent predictors affecting efficacy.

Received January 4, 2010; revised manuscript received May 12, 2010; accepted May 13, 2010; released online July 29, 2010 Time for primary review: 14 days

Cardiovascular Center and Department of Internal Medicine, Seoul National University Hospital, Seoul, South Korea

Mailing address: Hyo-Soo Kim, MD, PhD, Department of Internal Medicine, Seoul National University College of Medicine, 28 Yongon-dong Chongno-gu, Seoul 110-744, South Korea. E-mail: hyosoo@snu.ac.kr or Tae-Jin Youn, MD, PhD, Department of Internal Medicine, Seoul National University College of Medicine, Seoul, South Korea. E-mail: ytjmd@ snubh.org

ISSN-1346-9843 doi:10.1253/circj.CJ-09-1041

All rights are reserved to the Japanese Circulation Society. For permissions, please e-mail: cj@j-circ.or.jp 


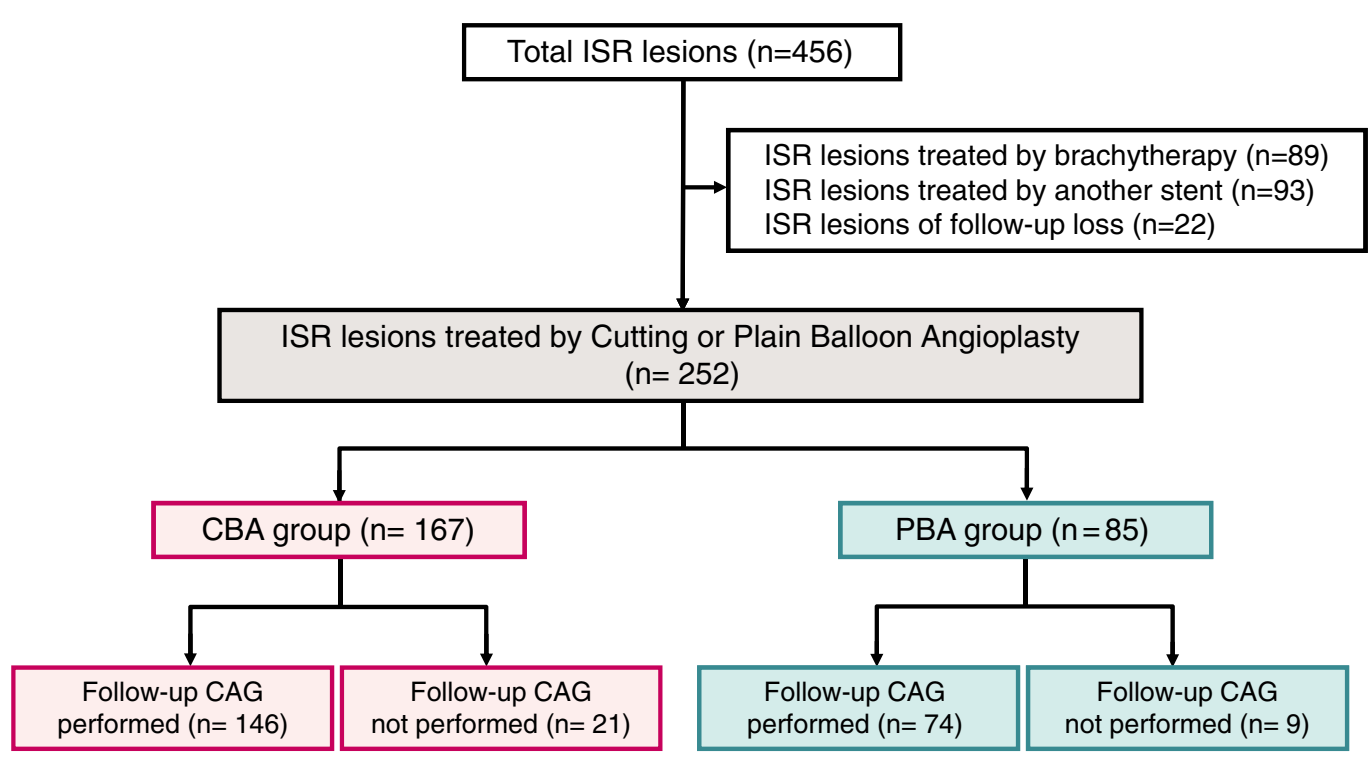

Figure 1. Flow chart of the present study. CAG, coronary angiography; CBA, cutting balloon angioplasty; ISR, in-stent restenosis; PBA, plain balloon angioplasty.

\section{Methods}

\section{Patient Population}

We analyzed all patients with ISR treated with either CBA or PBA in the Seoul National University Hospital ISR cohort from July 2002 to December 2007. We excluded ISR treated by brachytherapy, atherectomy, or additional stent implantation. All enrolled patients presented with recurrent angina and/or angiographic evidence of ISR. All the DES-ISR lesions were with either sirolimus-eluting stents $\left(\right.$ Cypher $^{\circledR}$, Cordis, Miami Lakes, FL, USA) or paclitaxel-eluting stents (Taxus ${ }^{\circledR}$, Boston Scientific, Natick, MA, USA).

ISR was defined as significant luminal narrowing [ $>50 \%$ diameter stenosis (DS) by quantitative coronary angiography (QCA)] within the stented segment or within $5 \mathrm{~mm}$ of the stent's edges. The lesions were categorized as focal $(<10 \mathrm{~mm}$ in length) or diffuse ( $\geq 10 \mathrm{~mm}$ in length or total occlusion). The ISR lesions were grouped according to the type of balloon used to treat them; 'CBA group' or 'PBA group'.

\section{Procedural Details}

All patients were pretreated with aspirin. Patients who were not already on a maintenance dose of clopidogrel after the initial stent implantation were also administered a loading dose of $300 \mathrm{mg}$ of clopidogrel prior to the procedure. After intervention, patients received aspirin (100 mg daily) indefinitely and clopidogrel ( $75 \mathrm{mg}$ daily) for at least 3 months.

During the coronary intervention, patients received anticoagulation with unfractionated heparin (bolus of $40 \mathrm{U} / \mathrm{kg}$ and additional heparin to achieve activated clotting time of 250-300s). The use of glycoprotein IIb/IIIa inhibitors and the choice of treatment modality (CBA or PBA) were at the operator's discretion.

Plain Balloon Angioplasty Noncompliant or semi-compliant plain balloons were used that matched the size of the final balloon selected at the time of stent implantation. To minimize residual stenosis, multiple high-pressure inflations
(>12 atm) were usually performed, because the manufacturers commonly recommended 12-18 atm.

Cutting Balloon Angioplasty Cutting balloons of $10-15 \mathrm{~mm}$ in length with the same or $0.25-0.5 \mathrm{~mm}$ larger diameter than the reference vessel were inflated several times at the lesion site to the recommended maximal pressure of 8-12atm. When the result was suboptimal, we changed the initial cutting balloon to a still larger one (by $0.25-0.5 \mathrm{~mm}$ ) because of its lower burst pressure than a plain balloon. In cases of unsuccessful passage, predilatation using a small plain balloon was performed.

Balloon angioplasty was regarded as successful if the final residual stenosis was less than $30 \%$, with Thrombolysis In Myocardial Infarction grade 3 flow.

\section{Endpoints and Clinical Definitions}

Angiographic follow-up was recommended to all patients at 6 months after the procedure or earlier if clinically indicated. The primary angiographic outcome was repeat-ISR (recurrence of $>50 \%$ DS) and in-stent late luminal loss (LLL) as assessed by QCA at 6 months. The secondary outcomes were cardiac death, non-fatal myocardial infarction (MI), and target lesion revascularization (TLR) up to 12 months. All deaths were considered cardiac unless the specific cause of death was documented. MI was defined as an increased creatine kinase-MB level $\geq 3$-fold the upper normal limit with and without development of new ECG changes compatible with MI. TLR was defined as any revascularization because of in-stent recurrence including the 5-mm segments adjacent to the stent. STH included definite and probable cases as defined by the Academic Research Consortium. ${ }^{18}$

Clinical follow-up data were obtained from the hospital's electronic medical records in the outpatient office visit, as well as from the re-admissions for 12 months after the treatment. Clinical events such as death, MI, and TLR were adjudicated by an independent researcher who was unaware of the assigned treatment. 


\begin{tabular}{lccc}
\hline Table 1. Baseline Characteristics of CBA and PBA Groups & & \\
& CBA & PBA & P value \\
Patients $(n)$ & 149 & 75 & \\
Clinical characteristics & & & \\
Age, years & $63.9 \pm 10.1$ & $63.8 \pm 8.5$ & 0.96 \\
Male & $105(70.5 \%)$ & $46(61.3 \%)$ & 0.22 \\
Hypertension & $89(59.7 \%)$ & $43(57.3 \%)$ & 0.73 \\
Diabetes & $65(43.6 \%)$ & $30(40.0 \%)$ & 0.60 \\
Hyperlipidemia & $66(44.3 \%)$ & $32(42.7 \%)$ & 0.94 \\
Smoking & $71(47.7 \%)$ & $30(40.0 \%)$ & 0.31 \\
LVEF, \% & $57.0 \pm 11.1$ & $57.3 \pm 9.8$ & 0.80 \\
Previous MI & $42(28.2 \%)$ & $15(20.0 \%)$ & 0.20 \\
Previous coronary bypass surgery & $10(6.7 \%)$ & $9(12.0 \%)$ & 0.16 \\
ACS & $27(18.1 \%)$ & $16(21.3 \%)$ & 0.57 \\
Lesion and procedural characteristics & & & \\
Lesions ( $)$ & 167 & 85 & 0.54 \\
LAD lesion & $80(47.9 \%)$ & $43(50.6 \%)$ & 0.82 \\
DES-ISR & $105(62.9 \%)$ & $52(61.2 \%)$ & 0.82 \\
BMS-ISR & $62(37.1 \%)$ & $33(38.8 \%)$ & 0.56 \\
Stent diameter, mm & $3.05 \pm 0.44$ & $3.02 \pm 0.38$ & 0.76 \\
Stent length per lesion, mm & $31.8 \pm 17.9$ & $31.0 \pm 18.1$ & 0.06 \\
Focal-ISR (<10mm) & $95(56.9 \%)$ & $59(69.4 \%)$ & 0.06 \\
Diffuse-ISR $(\geq 10 \mathrm{~mm})$ & $72(43.1 \%)$ & $26(30.6 \%)$ & 0.07 \\
Pre-treatment diameter stenosis, \% & $67.2 \pm 13.2$ & $71.4 \pm 18.2$ & 0.86 \\
Maximal balloon size, mm & $3.03 \pm 0.35$ & $3.04 \pm 0.44$ & \\
\hline
\end{tabular}

Clinical characteristics were evaluated on a per patient basis, and those of lesion or procedure on a per lesion basis. Data are number $(\%)$ or mean \pm standard deviation.

CBA, cutting balloon angioplasty; PBA, plain balloon angioplasty; LVEF, left ventricular ejection fraction; MI, myocardial infarction; ACS, acute coronary syndrome; LAD, left anterior descending coronary artery; DES, drug-eluting stent; ISR, in-stent restenosis; BMS, bare metal stent.

\begin{tabular}{|lccc|}
\hline \multicolumn{2}{|c|}{ Table 2. Angiographic and Clinical Outcomes for the Treatment of Total ISR } & PBA & P value \\
Patients (n) & CBA & 75 & \\
Lesions (n) & 149 & 85 & 0.97 \\
QCA data at 6-month follow-up & 167 & $\mathrm{n}=74(87.0 \%)$ & 0.46 \\
Lesion length, mm & $\mathrm{n}=146(87.4 \%)$ & $14.6 \pm 8.6$ & \\
RVD, mm & $15.7 \pm 9.6$ & & 0.76 \\
Pretreatment & & $2.57 \pm 0.53$ & 0.38 \\
Post-treatment & $2.55 \pm 0.58$ & $2.61 \pm 0.57$ & 0.60 \\
Follow-up & $2.68 \pm 0.53$ & $2.58 \pm 0.40$ & \\
MLD, mm & $2.53 \pm 0.49$ & & 0.32 \\
Pretreatment & & $0.75 \pm 0.60$ & 0.35 \\
Post-treatment & $0.82 \pm 0.38$ & $1.95 \pm 0.47$ & 0.20 \\
Follow-up & $2.01 \pm 0.46$ & $1.48 \pm 0.42$ & \\
DS, \% & $1.57 \pm 0.59$ & & 0.07 \\
Pretreatment & & $71.4 \pm 18.2$ & 0.70 \\
Post-treatment & $67.2 \pm 13.2$ & $25.0 \pm 13.3$ & 0.23 \\
Follow-up & $24.2 \pm 12.1$ & $37.7 \pm 12.5$ & 0.92 \\
Late loss, mm & $39.7 \pm 20.0$ & $0.61 \pm 0.47$ & 0.90 \\
Repeat-ISR, \% & $0.62 \pm 0.60$ & $28(37.8 \%)$ & 1.00 \\
Clinical follow-up for 12 months & $54(37.0 \%)$ & $\mathrm{n}=75(100 \%)$ & 0.50 \\
TLR & $\mathrm{n}=149(100 \%)$ & $19(22.4 \%)$ & 0.03 \\
MI & $31(18.3 \%)$ & 0 & 0.55 \\
Cardiac death & $9(6.0 \%)$ & 0 & \\
\hline
\end{tabular}

Late loss, repeat-ISR, and TLR were evaluated on a per lesion basis, whereas $\mathrm{Ml}$ and cardiac death were on a per patient basis. Data are number $(\%)$ or mean \pm standard deviation.

QCA, quantitative coronary angiography; RVD, reference vessel diameter; MLD, minimal luminal diameter; DS, diameter stenosis; TLR, target lesion revascularization. Other abbreviations see in Table 1. 


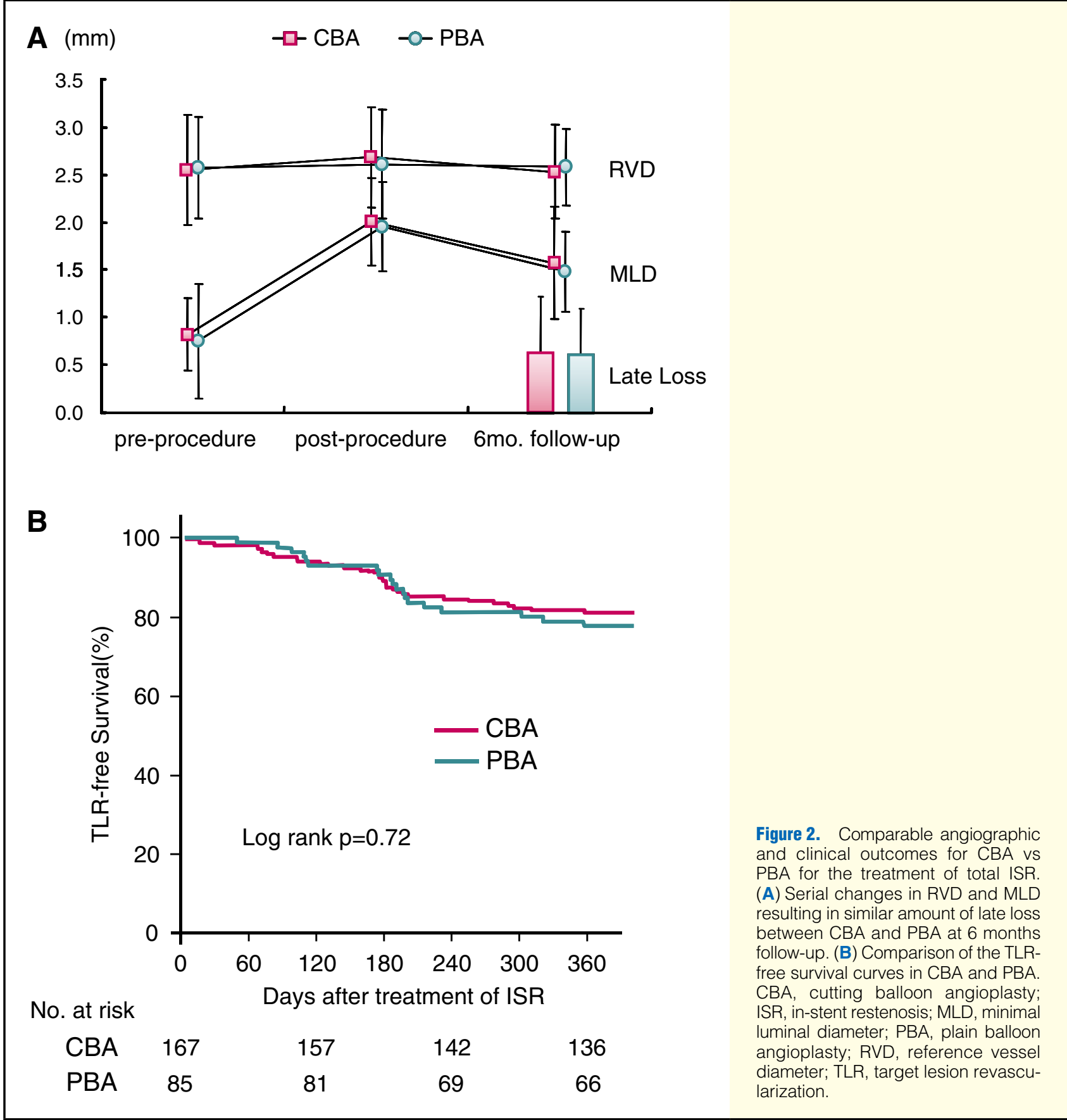

\section{Quantitative Coronary Angiography}

For each lesion, the single angiogram demonstrating the most severe degree of stenosis was used for QCA. All angiograms were analyzed by 2 independent operators who used a validated edge detection system, CAAS QCA for Research 2.0.1 (Pie Medical Imaging, Maastricht, the Netherlands). Intracoronary nitroglycerin $(200 \mu \mathrm{g})$ was administered before each angiogram was recorded. The contrast-filled catheter was used for calibration. QCA was performed before and after the procedure and at follow-up in the same projection at end-diastole. Lesion length was measured as the distance from shoulder to shoulder. Reference vessel diameter (RVD), minimal lumen diameter (MLD) and percentage DS were measured at baseline, post-procedure and follow-up. Repeat-
ISR was defined as DS $>50 \%$ by QCA within the initially stented segment (intra-stent and within $5 \mathrm{~mm}$ of the stent's edges) on the follow-up angiogram. LLL was defined as the difference between follow-up and post-procedural MLDs.

\section{Statistical Analysis}

Continuous variables are presented as mean \pm standard deviation and compared using Student's t-test. The results for categorical variables are described as percentages, and the $\chi^{2}$ test or Fisher's exact test was used to compare them when appropriate. TLR-free survival was estimated by Kaplan-Meier analysis and compared with the log-rank test. Univariate and multivariate analyses were performed using logistic regression with the clinical and lesion-related variables used to 


\begin{tabular}{|c|c|c|c|c|c|c|c|c|c|}
\hline & \multicolumn{9}{|c|}{ CBA vs PBA } \\
\hline & \multicolumn{3}{|c|}{ Late loss } & \multicolumn{3}{|c|}{ Repeated-ISR } & \multicolumn{3}{|c|}{ TLR } \\
\hline & $\beta$ & $95 \% \mathrm{Cl}$ & $P$ value & OR & $95 \% \mathrm{Cl}$ & $P$ value & OR & $95 \% \mathrm{Cl}$ & $P$ value \\
\hline Univariate & -0.01 & $-0.18-0.16$ & 0.92 & 0.97 & $0.55-1.71$ & 0.91 & 0.83 & $0.46-1.47$ & 0.51 \\
\hline Multivariate & -0.03 & $-0.22-0.15$ & 0.72 & 1.22 & $0.58-2.57$ & 0.60 & 1.20 & $0.58-2.52$ & 0.62 \\
\hline$P S 1^{*}$ & -0.07 & $-0.25-0.12$ & 0.48 & 1.25 & $0.64-2.46$ & 0.51 & 1.22 & $0.61-2.45$ & 0.58 \\
\hline $\mathrm{PS}^{*}$ & -0.04 & $-0.22-0.15$ & 0.70 & 1.24 & $0.59-2.61$ & 0.57 & 1.20 & $0.57-2.50$ & 0.63 \\
\hline
\end{tabular}

${ }^{*}$ PS1 and PS2 represent propensity score-adjusted logistic regression analyses 1 and 2; the first model included only 3 variables of treatment modality (CBA vs PBA), propensity score and post-treatment MLD whereas the second model included all the variables in Table 3 as well as propensity score.

$\mathrm{Cl}$, confidence interval; OR, odds ratio. Other abbreviations see in Tables 1,2.
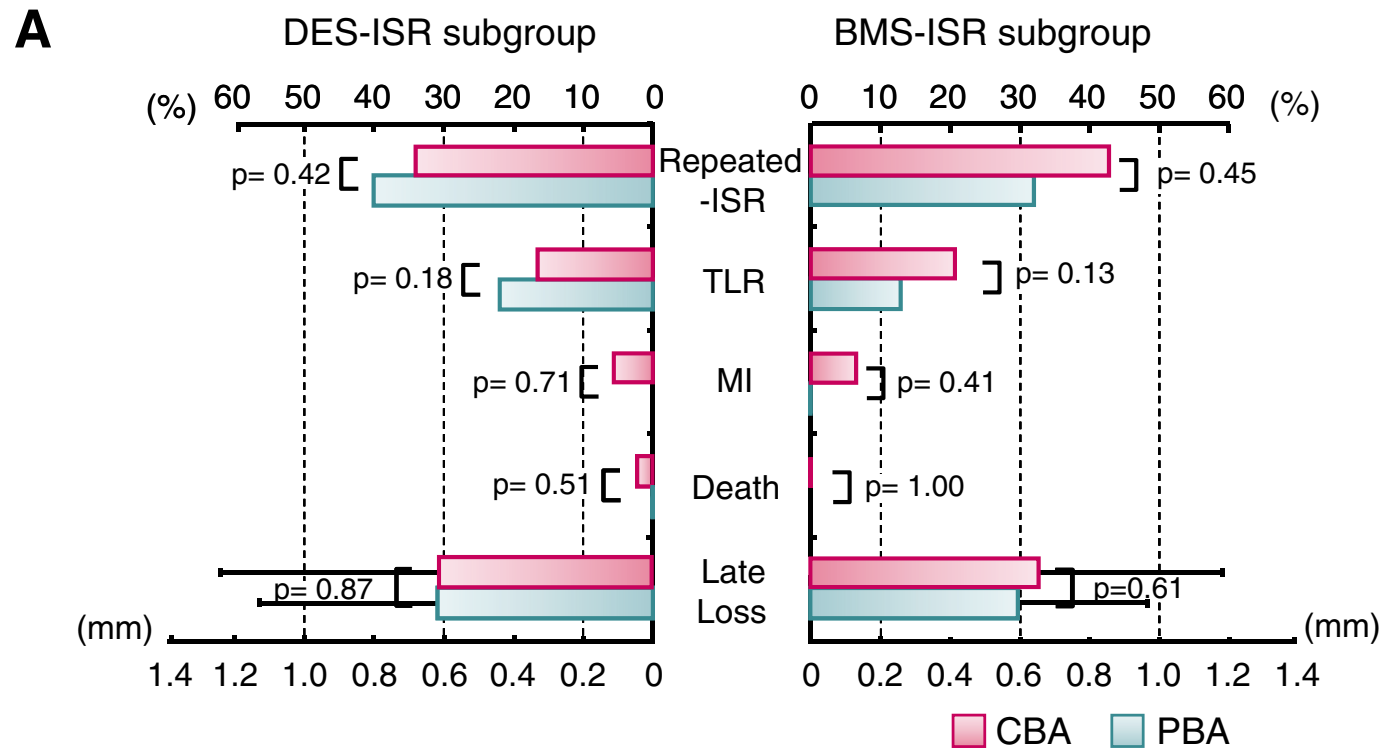

B

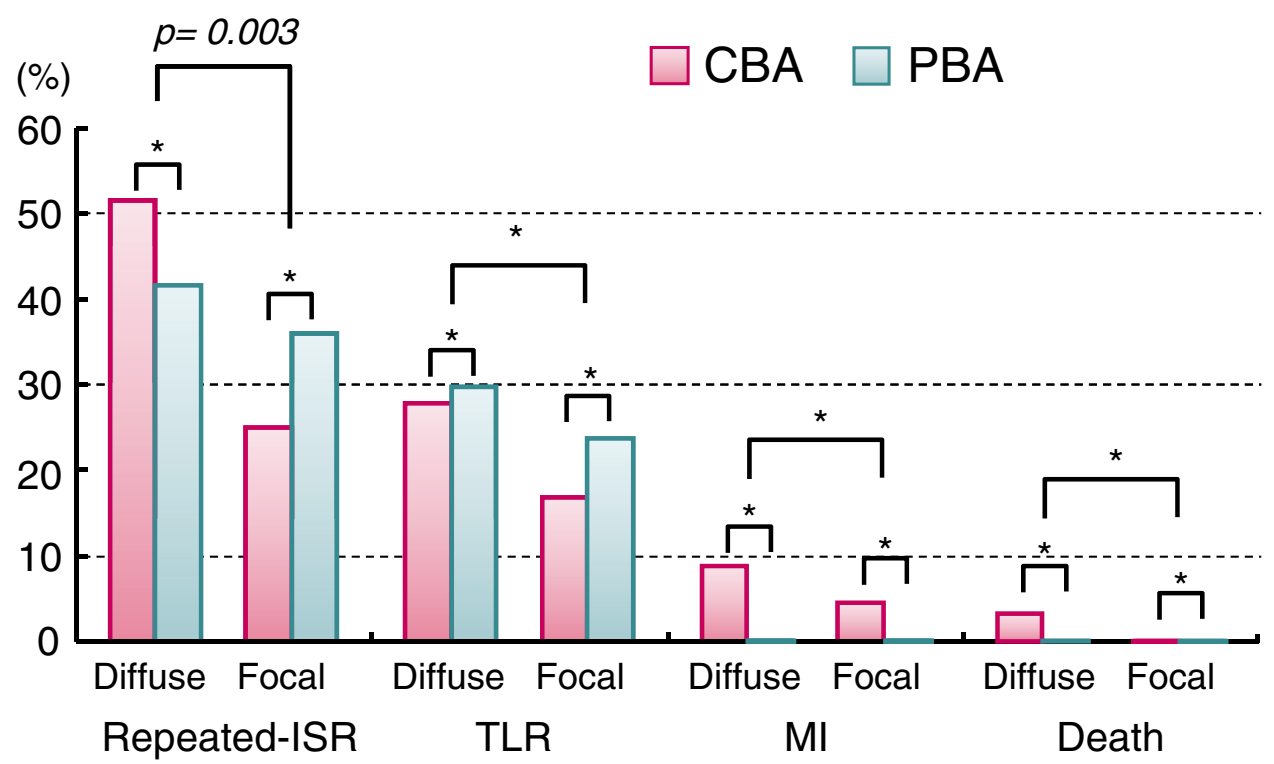

Figure 3. Comparable efficacy between CBA and PBA in various subgroups: DES-ISR and BMS-ISR (A), diffuse-ISR and focal-ISR (B). Diffuseness of ISR lesions rather than type of balloon (cutting vs plain) predicted repeat-ISR. BMS, bare metal stent; CBA, cutting balloon angioplasty; DES, drug-eluting stent; Diffuse, diffuse-ISR; Focal, focal-ISR; ISR, in-stent restenosis; MI, myocardial infarction; PBA, plain balloon angioplasty; TLR, target lesion revascularization. ${ }^{*} \mathrm{P}=$ not significant (NS). 


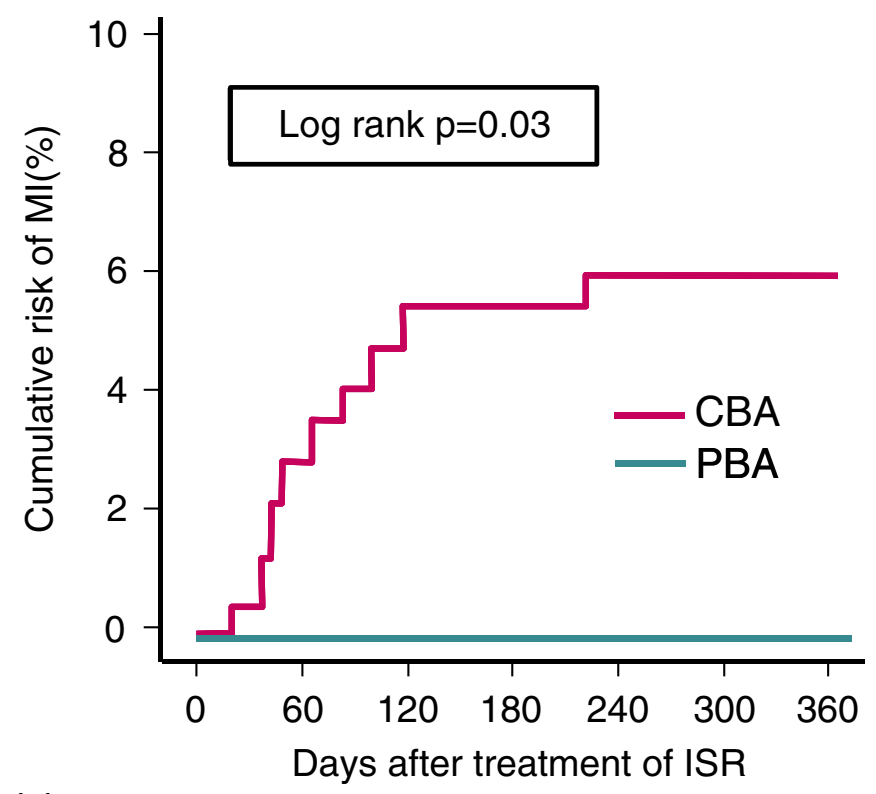

No. at risk

CBA 149

141

75
140

75
Figure 4. Higher rate of target lesion-related $\mathrm{MI}$ in CBA vs PBA after the treatment of total ISR. CBA, cutting balloon angioplasty; ISR, in-stent restenosis; MI, myocardial infarction; PBA, plain balloon angioplasty. evaluate independent predictors of repeat-ISR.

To adjust for the difference in the baseline characteristics of the 2 groups (CBA vs PBA), the propensity score as a probability of the use of ' $\mathrm{CBA}$ ' for each patient was calculated using a non-parsimonious multivariate logistic regression model in which baseline parameters (Table 4), other than post-treatment MLD, were incorporated. Because the propensity score model is used to minimize selection bias at the time when a specific treatment is chosen, the factors specified after determination of the type of balloon (eg, post-treatment MLD) were excluded from the calculation. The c-statistic of the propensity score model was 0.68 . Next, the angiographic and clinical outcomes according to the type of balloon were compared using 2 models of propensity score-adjusted logistic regression analysis; the first model included only 3 variables of treatment modality (CBA vs PBA), propensity score and post-treatment MLD whereas the second model included all the variables in Table 4, as well as the propensity score.

All statistical analyses were performed with SPSS software, version 17 (SPSS Inc, Chicago, IL, USA). Significance of all tests was defined at the $\mathrm{P}<0.05$ level. All reported $\mathrm{P}$-values are 2-tailed.

\section{Results}

Patient Population and Baseline Lesion Characteristics

Among 456 ISR lesions in 401 patients from the Seoul National University Hospital ISR cohort, we excluded 89 lesions in 74 patients and 93 cases in 87 patients treated by brachytherapy and additional stent implantation, respectively. Patients without follow-up (22 lesions in 16 patients) were also excluded. Finally, a total of 252 ISR lesions in 224 patients were included in the analysis (Figure 1). CBA was used in 167 lesions and PBA in 85 lesions. There was no significant difference in the baseline clinical characteristics of the 2 angioplasty groups, which were evaluated on a per patient basis, except for a trend toward a higher rate of previous coronary bypass surgery in the PBA arm (Table 1).

Both treatment groups also showed similar baseline lesion and procedural characteristics, which were analyzed on a per lesion basis, such as initial stent diameter $(\mathrm{P}=0.56)$, stent length $(\mathrm{P}=0.76)$ and the distribution of initial stent type; $\mathrm{CBA}$ was used for 105 DES-ISR and 62 BMS-ISR lesions, whereas it was PBA for 52 DES-ISR and 33 BMS-ISR lesions $(\mathrm{P}=$ 0.82). The maximal balloon size in the CBA group achieved during the procedure was also similar to that in the PBA group $(\mathrm{P}=0.86)$, despite the different inflation strategies. There was a trend toward more frequent use of CBA over PBA in diffuse-ISR lesions $(\mathrm{P}=0.06)$. On the other hand, pretreatment DS tended to be more severe in the PBA arm than in the CBA $(\mathrm{P}=0.07)$.

\section{Efficacy of CBA vs PBA for the Treatment of Total ISR Lesions}

Table 2 shows the angiographic and clinical outcomes. The rate of angiographic follow-up was nearly equal (87.4\% vs $87.0 \%, \mathrm{P}=0.97$ ) in both groups and clinical follow-up was completed in all of the patients of both arms. The incidence of repeat-ISR ( $37.0 \%$ vs $37.8 \%, \mathrm{P}=0.90)$ and LLL $(0.62 \pm 0.60$ vs $0.61 \pm 0.47 \mathrm{~mm}, \mathrm{P}=0.92$ ) at 6 months was similar between both groups (Figure 2A). At 12-month clinical follow-up, both treatment groups showed no significant difference in the rate of TLR $(18.3 \%$ vs $22.4 \%, \mathrm{P}=0.50)$ or cardiac death (Table 3). Kaplan-Meier TLR-free survival curves revealed a similar pattern between both arms $(81.7 \%$ vs $77.6 \%, \mathrm{P}=0.72$ by log-rank test) (Figure 2B).

This comparable efficacy between CBA and PBA was consistently maintained in various subgroups divided by initial stent type (DES-ISR vs BMS-ISR) (Figure 3A), pattern of ISR (diffuse-ISR vs focal-ISR) (Figure 3B), and other clini- 


\begin{tabular}{|c|c|c|c|c|}
\hline & \multicolumn{2}{|c|}{ Univariate analysis } & \multicolumn{2}{|c|}{ Multivariate analysis } \\
\hline & OR (95\%Cl) & $P$ value & OR (95\%Cl) & $P$ value \\
\hline Age $\geq 65$ years & $1.52(0.84-2.63)$ & 0.17 & $1.18(0.57-2.60)$ & 0.68 \\
\hline Male & $1.42(0.70-2.40)$ & 0.38 & $1.17(0.57-2.96)$ & 0.81 \\
\hline Diabetes & $1.11(0.78-1.09)$ & 0.76 & $1.43(0.75-2.96)$ & 0.41 \\
\hline Hypertension & $1.64(0.95-3.01)$ & 0.08 & $2.56(1.10-6.21)$ & 0.04 \\
\hline Smoking & $1.66(0.95-2.94)$ & 0.10 & $2.13(0.85-4.87)$ & 0.12 \\
\hline Dyslipidemia & $1.26(0.68-2.08)$ & 0.61 & $1.57(0.75-3.32)$ & 0.27 \\
\hline Creatinine $\geq 1.5 \mathrm{mg} / \mathrm{dl}$ & $2.10(0.92-4.85)$ & 0.09 & $1.32(0.43-4.02)$ & 0.66 \\
\hline Time to last stent implantation & $1.02(0.99-1.05)$ & 0.17 & $1.07(0.98-1.14)$ & 0.07 \\
\hline Previous MI & $1.05(0.56-1.95)$ & 0.90 & $1.31(0.52-3.22)$ & 0.56 \\
\hline Previous CABG & $1.27(0.56-3.49)$ & 0.66 & $1.38(0.56-5.43)$ & 0.36 \\
\hline LVEF $<40 \%$ & $1.51(0.46-4.27)$ & 0.26 & $1.26(0.26-5.71)$ & 0.78 \\
\hline ACS & $1.34(0.65-2.80)$ & 0.42 & $1.37(0.51-3.52)$ & 0.54 \\
\hline LAD lesion & $1.12(0.65-1.89)$ & 0.77 & $1.25(0.64-2.51)$ & 0.71 \\
\hline Stent diameter $<3 \mathrm{~mm}$ & $1.08(0.63-1.98)$ & 0.76 & $1.18(0.53-2.48)$ & 0.65 \\
\hline Stent length $\geq 30 \mathrm{~mm}$ & $0.95(0.53-1.52)$ & 0.81 & $0.69(0.35-1.39)$ & 0.41 \\
\hline Diffuse ISR & $2.48(1.41-4.39)$ & $<0.01$ & $2.15(1.03-4.46)$ & 0.04 \\
\hline CBA over PBA & $0.97(0.55-1.71)$ & 0.91 & $1.22(0.58-2.57)$ & 0.60 \\
\hline Pretreatment MLD $<0.8 \mathrm{~mm}^{*}$ & $2.95(1.63-5.35)$ & $<0.01$ & $2.58(1.20-5.61)$ & 0.03 \\
\hline Post-treatment MLD $<2.0 \mathrm{~mm}^{*}$ & $1.54(0.85-2.78)$ & 0.20 & $1.11(0.54-2.35)$ & 0.81 \\
\hline
\end{tabular}

${ }^{*}$ Mean value of pre-PCI MLD in all ISR lesions was $0.80 \mathrm{~mm}$ whereas that of post-PCI MLD was $0.20 \mathrm{~mm}$.

CABG, coronary artery bypass graft surgery. Other abbreviations see in Tables $1-3$.

cally relevant parameters (Figure S1).

Moreover, even after adjustment for differences in the baseline characteristics of both treatment groups using 2 models of propensity score-adjusted logistic regression analysis, no significant differences were observed regarding the angiographic and clinical endpoints (Table 3).

However, the rate of target lesion-related MI was significantly higher in the CBA group (6.0\% vs $0 \%, \mathrm{P}=0.03)$. During the 12-month follow-up, a total of 11 patients experienced MI: 2 cases occurred in a non-target lesion-related territory and interestingly, 9 cases of target lesion-related infarctions developed only in the CBA group because of STH $(n=8)$ or loss of a side branch in the stented segment $(n=1)$. Moreover, most of these patients visited the emergency department early after discharge prior to the scheduled follow-up (Figure 4). In the PBA group, however, there were no cases of MI or cardiac death (detailed information is shown in Table S1).

\section{Independent Predictors of Repeat-ISR After Angioplasty}

As shown in Table 4, diffuse-ISR and smaller pretreatment MLD were univariate predictors of repeat-ISR. Even in the multivariate analysis, these 2 parameters remained as independent predictors (odds ratio (OR) $2.15,95 \%$ confidence interval (CI) 1.03-4.46, P=0.04; OR 2.58, 95\%CI 1.20-5.61, $\mathrm{P}=0.03$, for diffuse-ISR and smaller pretreatment MLD, respectively). The only clinical predictor was hypertension, and time from stent implantation to ISR showed a marginal significance $(\mathrm{P}=0.07)$. Other factors, such as diabetes, left ventricular dysfunction, and initial stent length, were not independently associated with it.

\section{Post-Hoc Analysis of ISR Lesions Treated by Additional Stenting Following BA}

According to some previous reports, ${ }^{15,17} \mathrm{CBA}$ is more effective than PBA in minimizing balloon slippage and slippage-induced dissection. Therefore, we performed a post-hoc analysis of the rate of these events not only in the balloon treated group $(n=252)$ but also in the stented group $(n=93)$, which were excluded from the analysis at first (Figure 1). In the stented lesions, a total 8 patients with ISR affecting only the intra-stent site were found to have undergone additional stenting because of a balloon slippage-induced significant dissection (type D-F): 3 after CBA vs 5 after PBA. When these cases $(n=8)$ were incorporated into the original data $(n=252)$, indeed we did observe a trend toward less need for additional stent implantation required by dissection in the CBA vs PBA group (1.7\% vs 5.4\%, $\mathrm{P}=0.13$ ) (Table S2).

\section{Discussion}

\section{Comparable Efficacy Between PBA and CBA for ISR}

Initial experience with CBA reported more favorable results compared with PBA in the treatment of de novo stenosis, ${ }^{14,19}$ but that efficacy could not be confirmed in a multicenter randomized trial. ${ }^{16}$ There was no difference in the restenosis rate at 6 months $(31.4 \%$ vs $30.4 \%, P=0.75)$. Similarly, CBA has failed to demonstrate superiority over PBA for BMSISR. In an initial retrospective study, ${ }^{15} \mathrm{CBA}$ showed a lower incidence of repeat-ISR and TLR vs PBA. In the larger randomized RESCUT trial, ${ }^{17}$ however, CBA did not reduce the rate of either repeat-ISR $(29.8 \%$ vs $31.4 \%, \mathrm{P}=0.82)$ or major adverse cardiac events $(16.4 \%$ vs $15.4 \%, \mathrm{P}=0.79)$. Interestingly, our study also showed no difference between these for the treatment of DES-ISR, as well as BMS-ISR, with regard to the angiographic and clinical endpoints. Furthermore, this comparable efficacy between both methods was consistently maintained in various subgroup analyses and even after adjustment using the propensity score.

Differences in the Mechanisms of Action of PBA vs CBA

There are some possible explanations for the lack of benefit of CBA over PBA. In general, the severity of restenosis is 
related to the degree of vessel trauma. ${ }^{20}$ For de novo stenosis, CBA theoretically might have the advantage of lesser vascular injury vs PBA, because it can exert a relatively even degree of stretching force against the vessel as a result of several incisions made at a regular distance, thereby avoiding localized overstretch in weaker areas, ${ }^{16,21}$ which would occur in PBA. However, in cases of ISR, uneven stretch of the coronary artery could be minimized with the help of stent struts, irrespective of the type of balloon. Therefore, the potential benefit of CBA over PBA could be attenuated in ISR treatment. Secondly, in DES-ISR, as well as in BMSISR, the requirement for repeat-ISR is more greatly affected by the diffuseness of the initial ISR $^{22,23}$ than by the type of treatment (cutting or plain balloon).

\section{Safer and More Practical Profiles of PBA Compared With CBA} We could not find superiority of CBA over PBA in the treatment of DES-ISR or BMS-ISR. On the contrary, the incidence of target lesion-related MI was more frequent and actually occurred exclusively in the CBA group. Moreover, most MI occurred in the early phase after treatment before routine follow-up (Figure 4). These unfavorable results for CBA were also found in other trials, ${ }^{17,24}$ where CBA vs PBA showed a trend towards an increase in periprocedural MI (within 30 days) without reducing restenosis for 1 year. The reason for the higher incidence of MI after CBA could be related to CBA causing a wider extent of injury in the ISR lesions, with the sharp microblades more severely denuding the re-endothelialized stent struts, exposing a larger amount of thrombogenic material and provoking stronger activation of platelets, coagulation, and inflammation. It is premature, however, to say definitely that CBA raises the risk of MI vs PBA in the treatment of ISR based upon this retrospective analysis, because of possible confounders, but it might be reasonable at least to pay more attention to dual anti-platelet therapy after CBA for ISR patients with many risk factors of STH.

On the other hand, the more favorable profile of plain balloons guarantees better ability to cross tight ISR lesions compared with the bulky cutting balloon, ${ }^{17}$ as shown in the RESCUT trial (failure to cross the lesions; CBA 5.2\% vs PBA $0 \%, \mathrm{P}<0.01)$. Not only CBA but also another DES implantation is often used for treatment of DES-ISR. However, the risk of DES thrombosis could be significantly elevated by this approach, requiring much longer duration of dual antiplatelet therapy. ${ }^{9-11,23}$ Taking all the findings together, PBA could be considered as a safe, practical, and effective treatment modality for DES-ISR, with $37.8 \%$ repeat-ISR and $22.4 \%$ TLR in our study, which seemed acceptable compared with the outcomes from other trials. ${ }^{11,15,23}$

\section{Importance of Parameters Associated With Plaque Burden of ISR Lesions}

Together with the diffuseness of the initial ISR lesion, smaller pretreatment MLD and hypertension were also identified as independent predictors. The first 2 parameters (diffuseness and smaller pretreatment MLD) might represent a heavier plaque burden of the ISR lesion. Therefore, continuous efforts to reduce plaque volume with effective medical treatment might also be important for optimal management of ISR.

\section{Study Limitations}

This study has the limitations inherent to a retrospective observation study. Especially, the potential benefit of CBA to prevent dissections or crossover to stenting could be easily missed in our study because all patients who underwent repeated stenting were excluded from the initial analysis. On this important issue, however, we performed a post-hoc analysis, which indeed demonstrated a trend toward a reduction of slippage-induced significant dissection in the CBA group vs PBA. If we had incorporated cases of lesser degree of dissection as well, its potential benefit might have been more evident. Therefore, CBA may be particularly helpful for avoiding damage to important vessels adjacent to ISR lesions.

Secondly, this study was not a randomized controlled trial. However, we tried to compensate for this by using conventional multivariate and propensity score analyses comparing the 2 groups in terms of late loss, repeat-ISR, and TLR. Regarding MI, the OR was not reported as there was no event in the patients treated by PBA.

We also admit that the sample size is limited, which could be further complicated in the subgroups analyses. However, it is very difficult to collect DES-ISR cases treated by BA alone, especially in the DES era, because the rate of DESISR has become very low and many patients are treated by another strategy such as repeat stenting. To the best of our knowledge, our DES-ISR cohort treated by BA alone is 1 of the largest groups compared with other studies. ${ }^{6,7,26}$

Finally, in our study, a head-to-head comparison between PBA and additional DES for the treatment of DES-ISR was not performed. For this important subject, we are in the process of analyzing the cohort of patients treated by each treatment modality.

In conclusion, the efficacy of PBA was comparable to that of CBA for the treatment of DES-ISR, as well as BMSISR. PBA might be considered as a good option for ISR treatment, at least in focal lesions, which account for the majority of DES-ISR. More attention might be needed in the use of dual-antiplatelet therapy after CBA in ISR patients with many risk factors for STH. In addition, parameters related to the plaque volume of ISR lesions were independently associated with a higher rate of repeat-ISR.

\section{Acknowledgments}

This study was supported by a grant from the Innovative Research Institute for Cell Therapy, Seoul National University Hospital (A062260), and the Clinical Research Center for Ischemic Heart Disease, Seoul, Republic of Korea (0412-CR02-0704-0001) sponsored by the Ministry of Health \& Welfare, Republic of Korea.

\section{References}

1. Moses JW, Leon MB, Popma JJ, Fitzgerald PJ, Holmes DR, O'Shaughnessy C, et al. Sirolimus-eluting stents versus standard stents in patients with stenosis in a native coronary artery. $N$ Engl $J$ Med 2003; 349: 1315-1323.

2. Kataoka Y, Yasuda S, Morii I, Kawamura A, Miyazaki S. Improved long-term prognosis of elderly women in the era of sirolimus-eluting stents. Circ J 2009; 73: 1219-1227.

3. Rogers C, Edelman ER. Pushing drug-eluting stents into uncharted territory: Simpler than you think-more complex than you imagine. Circulation 2006; 113: 2262-2265.

4. Stone GW, Ellis SG, Cannon L, Mann JT, Greenberg JD, Spriggs D, et al. Comparison of a polymer-based paclitaxel-eluting stent with a bare metal stent in patients with complex coronary artery disease: A randomized controlled trial. JAMA 2005; 294: 1215-1223.

5. Cosgrave J, Agostoni P, Ge L, Iakovou I, Chieffo A, Biondi-Zoccai GG, et al. Clinical outcome following aleatory implantation of paclitaxel-eluting or sirolimus-eluting stents in complex coronary lesions. Am J Cardiol 2005; 96: 1663-1668.

6. Tagliareni F, La Manna A, Saia F, Marzocchi A, Tamburino C. Long-term clinical follow-up of drug-eluting stent restenosis treatment: Retrospective analysis from two high volume catheterisation laboratories. EuroIntervention 2010; 5: 703-708.

7. Kitahara H, Kobayashi Y, Takebayashi H, Fujimoto Y, Nakamura Y, Kuroda N, et al. Re-restenosis and target lesion revascularization after treatment of sirolimus-eluting stent restenosis: Retrospective 
analysis of 4 Japanese hospitals. Circ $J$ 2009; 73: 867-871.

8. Kim KH, Kim DI, Kim IH, Kim JY, Han YC, Seol SH, et al Therapeutic strategy for in-stent restenosis based on the restenosis pattern after drug-eluting stent implantation. Korean Circ J 2009; 39: $408-413$

9. Camenzind E, Steg PG, Wijns W. Stent thrombosis late after implantation of first-generation drug-eluting stents: A cause for concern. Circulation 2007; 115: 1440-1455.

10. Lee SE, John SH, Lim JH, Rhew JY. Very late stent thrombosis associated with multiple stent fractures and peri-stent aneurysm formation after sirolimus-eluting stent implantation. Circ J 2008; 72: $1201-1204$.

11. Mishkel GJ, Moore AL, Markwell S, Shelton MC, Shelton ME. Long-term outcomes after management of restenosis or thrombosis of drug-eluting stents. J Am Coll Cardiol 2007; 49: 181-184.

12. Oliver LN, Buttner PG, Hobson H, Golledge J. A meta-analysis of randomised controlled trials assessing drug-eluting stents and vascular brachytherapy in the treatment of coronary artery in-stent restenosis. Int J Cardiol 2008; 126: 216-223.

13. vom Dahl J, Dietz U, Haager PK, Silber S, Niccoli L, Buettner HJ, et al. Rotational atherectomy does not reduce recurrent in-stent restenosis: Results of the angioplasty versus rotational atherectomy for treatment of diffuse in-stent restenosis trial (ARTIST). Circulation 2002; 105: 583-588.

14. Izumi M, Tsuchikane E, Funamoto M, Kobayashi T, Sumitsuji S, Otsuji S, et al. Final results of the CAPAS trial. Am Heart J 2001; 142: $782-789$.

15. Adamian M, Colombo A, Briguori C, Nishida T, Marsico F, Di Mario C, et al. Cutting balloon angioplasty for the treatment of in-stent restenosis: A matched comparison with rotational atherectomy, additional stent implantation and balloon angioplasty. $J \mathrm{Am}$ Coll Cardiol 2001; 38: 672-679.

16. Mauri L, Bonan R, Weiner BH, Legrand V, Bassand JP, Popma JJ, et al. Cutting balloon angioplasty for the prevention of restenosis: Results of the Cutting Balloon Global Randomized Trial. Am J Cardiol 2002; 90: 1079-1083.

17. Albiero R, Silber S, Di Mario C, Cernigliaro C, Battaglia S, Reimers $\mathrm{B}$, et al. Cutting balloon versus conventional balloon angioplasty for the treatment of in-stent restenosis: Results of the restenosis cutting balloon evaluation trial (RESCUT). J Am Coll Cardiol 2004; 43: $943-949$.

18. Cutlip DE, Windecker S, Mehran R, Boam A, Cohen DJ, van Es $\mathrm{GA}$, et al. Academic Research Consortium: Clinical end points in coronary stent trials: A case for standardized definitions. Circulation 2007; 115: 2344-2351.
19. Kawaguchi K, Kondo T, Shumiya T, Uchikawa T, Suzuki T, Awaji $\mathrm{Y}$, et al. Reduction of early elastic recoil by cutting balloon angioplasty as compared to conventional balloon angioplasty. J Invasive Cardiol 2002; 14: 515-519.

20. Schwartz RS, Huber KC, Murphy JG, Edwards WD, Camrud AR, Vlietstra RE, et al. Restenosis and the proportional neointimal response to coronary artery injury: Results in a porcine model. $J \mathrm{Am}$ Coll Cardiol 1992; 19: 267-274.

21. Inoue T, Sakai Y, Hoshi K, Yaguchi I, Fujito T, Morooka S. Lower expression of neutrophil adhesion molecule indicates less vessel wall injury and might explain lower restenosis rate after cutting balloon angioplasty. Circulation 1998; 97: 2511-2518.

22. Cosgrave J, Melzi G, Biondi-Zoccai GG, Airoldi F, Chieffo A, Sangiorgi GM, et al. Drug-eluting stent restenosis: The pattern predicts the outcome. J Am Coll Cardiol 2006; 47: 2399-2404.

23. Mehran R, Dangas G, Abizaid AS, Mintz GS, Lansky AJ, Satler LF, et al. Angiographic patterns of in-stent restenosis: Classification and implications for long-term outcome. Circulation 1999; 100: $1872-1878$.

24. Bittl JA, Chew DP, Topol EJ, Kong DF, Califf RM. Meta-analysis of randomized trials of percutaneous transluminal coronary angioplasty versus atherectomy, cutting balloon atherotomy, or laser angioplasty. J Am Coll Cardiol 2004; 43: 936-942.

25. Alfonso F, Pérez-Vizcayno MJ, Hernández R, Bethencourt A, Martí V, López-Mínguez JR, et al. Long-term clinical benefit of sirolimus-eluting stents in patients with in-stent restenosis results of the RIBS-II (Restenosis Intra-stent: Balloon angioplasty vs elective sirolimus-eluting Stenting) study. J Am Coll Cardiol 2008; 52: $1621-1627$.

26. Colombo A, Latib A. Treatment of drug-eluting stent restenosis with another drug-eluting stent: Do not fail the second time. Rev Esp Cardiol 2008; 61: 1120-1122.

\section{Supplementary files}

Figure S1. Comparable 1-year TLR rate of PBA vs CBA in various subgroups divided according to multiple parameters.

Table S1. Detailed Information on the Cases of MI

Table S2. Post-Hoc Analysis of the Rate of Crossover to Additional Stenting Because of Significant Dissection or Suboptimal Result

Please find supplementary file(s);

http://dx.doi.org/10.1253/circj.CJ-09-1041 